\title{
How different is early-onset childhood disintegrative disorder from autistic disorder with speech loss?*
}

\author{
Hiroshi Kurita ${ }^{1,2 \#}$, Kanna Inoue ${ }^{3}$ \\ ${ }^{1}$ Department of Child Psychiatry, Zenkoku Ryoiku Sodan Center, Tokyo, Japan \\ ${ }^{2}$ Department of Mental Health, Graduate School of Medicine, Tokyo University, Tokyo, Japan \\ ${ }^{3}$ Department of Child Psychiatry, Nerima Welfare Center for Handicapped Persons, Tokyo, Japan \\ Email: ${ }^{*}$ hkurita@mvf.biglobe.ne.jp
}

Received 27 February 2013; revised 30 March 2013; accepted 9 April 2013

Copyright (C) 2013 Hiroshi Kurita, Kanna Inoue. This is an open access article distributed under the Creative Commons Attribution License, which permits unrestricted use, distribution, and reproduction in any medium, provided the original work is properly cited.

\section{ABSTRACT}

To examine the difference between early-onset $(<$ age 3) childhood disintegrative disorder (CDD) and autistic disorder with speech loss (ADSL), 8 children with early-onset CDD (mean age $=\mathbf{7 . 6}$ years, $\mathrm{SD}=\mathbf{3 . 8}$; 6 males) were compared with 92 age and gender-ratio comparable children with ADSL (mean age $=6.8$ years, $S D=4.1 ; 70$ males) on 24 variables not directly related to the key features of CDD (regression after normal development for at least the first 2 years after birth). Compared with the ADSL group, the earlyonset CDD group had a tendency to have a higher rate of a psychosocial event before speech loss (SL) (early-onset CDD, $\mathbf{7 5 . 0 \%}$ vs ADSL, 37.0\%, $p=0.057$; effect size $($ phi) $=0.211, p<0.05)$; a significantly higher rate of fearfulness during SL $(62.5 \%$ vs $4.3 \%$, p $=0.000 ;$ phi $=0.551, p<0.05) ;$ and a tendency to have a higher rate of epilepsy $(25.0 \%$ vs $3.3 \%, p=$ $0.050 ; \mathrm{phi}=0.271, \mathrm{p}<0.05$ ), a tendency to have a lower rate of the Childhood Autism Rating Scale-Tokyo Version (CARS-TV) total score $\geq \mathbf{3 0}(\mathbf{7 5 . 0 \%}$ vs $95.7 \%$, $\mathrm{p}=0.072 ;$ phi $=0.236, \mathrm{p}<0.05)$, and a significantly lower rate of CARS-TV item 2 (imitation) score $\geq 2$ $(50.0 \%$ vs $82.6 \%, p=0.049$; phi $=0.221, p<0.05)$ on the first visit. The two groups did not exhibit any significant difference in the other 19 variables. The findings of no significant difference in the great majority and a significant difference in the small minority of the 24 variables between the two groups support integrating CDD into regressive autism spectrum disorder and studying CDD as its prototypical form.

Keywords: Autistic Disorder; Autism Spectrum Disorder (ASD); Childhood Disintegrative Disorder;

\footnotetext{
"The authors declare that they have no conflict of interest

${ }^{\#}$ Corresponding author.
}

Diagnosis; Regression

\section{INTRODUCTION}

Childhood disintegrative disorder (CDD) originates from Dementia infantilis first reported by Heller [1] in 1908 in six infants who had displayed profound mental regression during the third and fourth years of life. CDD is a subtype of pervasive developmental disorders (PDDs) in DSM-IV [2] and ICD-10 [3], using similar diagnostic criteria. Its salient feature is a marked regression resulting in an autistic and intellectually disabled state after apparently normal development for at least the first 2 years after birth. Although studies on CDD are scarce due to its rarity (i.e. an estimated prevalence of 2.0/ 100,000 [4]), CDD is validated to a certain extent for its less favorable outcomes in childhood [5], adolescence, and adulthood [6,7], and in its higher incidence of epilepsy in childhood [8], compared with autistic disorder (AD). CDD is also distinct in its higher incidence of epilepsy, more pronounced stereotypical behavior, and a less uneven intellectual profile in childhood compared with $\mathrm{AD}$ with speech loss (SL) (ADSL), the type of AD that is closest to CDD [9].

DSM-5 [10] proposes to subsume CDD under the single category of autism spectrum disorder (ASD) together with three other DSM-IV PDDs (AD, Asperger's disorder and pervasive developmental disorder not otherwise specified (PDD-NOS)), with Rett's disorder (RD) excluded. However, it is important to further characterize $\mathrm{CDD}$, since many issues still remain unclear. One of them is the difference between early-onset $(<$ age 3$)$ CDD and AD with regression. Twenty-two of 57 (38.6\%) CDD cases in 14 studies [5,6,8,11-21] published in the last 25 years are early-onset, while $20 \%$ to $40 \%$ of $A D$ cases display regression [22-26].

Early-onset CDD cases satisfy not only CDD criteria 
A (normal for at least the first 2 years after birth), B (loss of acquired skills in at least two of the five areas) and $C$ (abnormalities in at least two of the three areas: social interaction impairment, communication impairment, and restricted/repetitive behavior/interests (RRBI)), but also $\mathrm{AD}$ criterion $\mathrm{B}$ (onset $<$ age 3 ) by definition of earlyonset (< age 3). If early-onset CDD cases satisfy CDD criterion $\mathrm{C}$ by showing abnormalities in all three areas instead of two, they are quite likely to also satisfy $A D$ criterion A (six or more symptom items in three areas, including at least two from social interaction impairment and one each from communication impairment and RRBI). In such cases, the conclusive CDD diagnosis requires that $\mathrm{CDD}$ criterion $\mathrm{D}$ (i.e. not better accounted for by another specific PDD or by schizophrenia) be met. Excluding RD, Asperger's disorder, PDD-NOS or schizophrenia is not difficult in such cases. However, a diagnosis of early-onset CDD by excluding AD may not be the ultimate diagnosis, since a diagnosis of $\mathrm{AD}$ is still possible in the event that $\mathrm{AD}$ criterion $\mathrm{C}$ (not better accounted for by RD or CDD) is considered to be met. This impasse remains in the absence of a rule by which it is determined that the diagnosis of $\mathrm{CDD}$ or $\mathrm{AD}$ takes priority in such cases. In addition, another rule is needed to diagnose early-onset CDD but not PDD-NOS in cases that satisfy the $\mathrm{CDD}$ criteria $\mathrm{A}$ to $\mathrm{C}$ and $\mathrm{AD}$ criterion $\mathrm{B}$, but does not meet the AD criterion A. However, DSMIV provides no such rules. Since we were aware of this shortcoming in CDD diagnosis in DSM-IV, we devised diagnostic criteria for early-onset CDD based on DSMIV CDD criteria as shown in Table 1 soon after the publication of DSM-IV to keep early-onset CDD as an integral part of CDD for CDD studies.

In this study, an effort was made to clarify the difference between early-onset $\mathrm{CDD}$ and regressive $\mathrm{AD}$ as represented by ADSL, through a comparison of clinical variables not directly related to the key features of CDD (regression after normal development for at least the first 2 years after birth).

\section{METHODS}

\subsection{Diagnostic Criteria for Early-Onset CDD}

Table 1 shows the diagnostic criteria for early-onset CDD. Criteria A to $\mathrm{C}$ are basically the same as those in DSM-IV CDD criteria, except for adding "before the age of 3" in criterion B to indicate the upper limit age at onset of regression (loss of acquired skills). Criterion D is revised extensively to allow a diagnosis of early-onset CDD by excluding other PDDs and schizophrenia. We used these criteria in this study.

\subsection{Subjects}

The subjects of this study were 8 children with early-
Table 1. Diagnostic criteria for early-onset childhood disintergrative disorder (CDD).

A. Apparently normal development for at least the first 2
years after birth.
B. Loss of acquired skills in at least two of the five areas
(language, social skills, bladder/bowel control, play and
motor skills) before the age of 3 .
C. Abnormalities in at least two of the three areas (social
interaction impairment, communication impairment and
restricted/repetitive behavior/interests).
D. With Rett's disorder, Asperger's disorder and schizophrenia
excluded, the diagnosis of early-onset CDD but not autistic disorder
or pervasive developmental disorder not otherwise specified in a
case that satisfies criteria A to C.

onset CDD (mean age 7.6 years, $\mathrm{SD}=3.8 ; 6$ males, 2 females) and 92 children with ADSL (mean age 6.8 years, $\mathrm{SD}=4.1 ; 70$ males, 22 females) not significantly different in age $(t(98)=0.522, p=0.603)$ and gender ratio $(\mathrm{p}=1.000)$ from the 8 children with early-onset CDD. They were collected in the period 1982-2011 as consecutive referrals to two leading facilities for developmental disorders in Tokyo (the authors' affiliations). In each facility, a clinical team of experienced clinicians (i.e. child psychiatrists, pediatric neurologists, psychologists and speech pathologists) lead by a child psychiatrist (the first author) diagnosed children according to the relevant ICD [3,27] and DSM criteria [2,28]. This retrospective chart review study, which was based on clinical data accumulated on patients in our clinics over the last 30 years, was one part of a comprehensive study of the prognoses of PDDs approved by the ethics committee of the Tokyo University Graduate School of Medicine.

\subsubsection{Early-Onset CDD}

Of the 8 children with early-onset CDD, 5 children who visited either of the two facilities after the publication of DSM-IV were diagnosed by the clinical teams as having early-onset CDD according to the criteria in Table 1 by consensus at the time of their first visit. The remaining 3 children, who visited one of the two facilities before the publication of DSM-IV, were diagnosed as having disintegrative psychosis based on ICD-9 [27]. The clinical teams reached a consensus from the details in the clinical records and diagnosed these 3 children as having early-onset CDD according to the criteria in Table 1. Experienced pediatric neurologists ruled out a neurological disease in the 8 children.

All of the 8 children satisfied criterion A, as indicated by having established normal social relations, at least one meaningful phrase, and pointing to objects of interest with the index finger before regression occurred at age 2 or later. They also satisfied criterion B (loss of acquired skills in at least two of the five areas before the age of 3: all had lost expressive language (area 1), social skills 
(area 2) and play (area 4); two had lost bladder control (area 3); and one had temporarily lost the ability to walk (area 5)) and criterion $\mathrm{C}$ by having had abnormalities in all of the three areas (social interaction impairment, communication impairment and RRBI). Although the 8 children also satisfied the DSM-IV AD criteria A and B, they were diagnosed as having early-onset CDD according to the criteria in Table 1.

\subsubsection{ADSL}

Of the 92 children with ADSL, 65 children who visited either of the two facilities after the publication of DSMIV were diagnosed as having $\mathrm{AD}$ by the clinical teams based on detailed evaluations performed at the first visit. The remaining 27 children who visited one of the two facilities before the publication of DSM-IV were diagnosed as having infantile autism according to DSM-III [28]. Based on the detailed clinical records, these 27 children were subsequently re-diagnosed as having DSM-IV AD by the clinical teams. Based on the detailed records on language development, the clinical teams judged the 92 children to have satisfied the SL criteria [22], modified by specifying the minimum number and duration of words expressed before SL, as follows: (a) an episode in which children lost the use of all meaningful words expressed spontaneously (i.e. at least two words spoken for one month or longer), and (b) after the loss of this vocabulary, remained mute for at least 6 months, regardless of whether they eventually recovered speech. Since SL also occurs in CDD as a cardinal symptom, in this study we used SL as a term to represent regression in CDD as well as ADSL.

Of the 92 children with ADSL, normal social relation, speaking at least one meaningful phrase, and pointing to objects of interest with the index finger before SL were reported in $14(15.2 \%), 4(4.3 \%)$, and $22(23.9 \%)$ children, respectively. However, none of the 92 children exhibited all of the three indicators of normal development before SL, and also normal development at age 2 or later so as to meet CDD criterion $\mathrm{A}$, unlike the 8 children with early-onset CDD.

\subsubsection{Assessment of Behavior and Development}

Experienced psychologists rated the 8 early-onset CDD children and 92 ADSL children with the Childhood Autism Rating Scale-Tokyo Version (CARS-TV) [29], a reliable and valid Japanese version of the Childhood Autism Rating Scale (CARS) [30] at the first visit. They also measured IQs of 67 of the children (3 early-onset CDD and 64 ADSL) with the Japanese version of the Stanford-Binet and developmental quotients (DQs) equivalent to IQs of 24 children (5 early-onset CDD and 19 ADSL), using either of the two standardized Japanese developmental scales $[31,32]$ at the first visit. These 91 children were classified into the two groups of "severe intellectual disability" (IQ/DQ < 35) and "other" (IQ/DQ $\geq 35$ ). Experienced psychologists classified the remaining 9 children with ADSL in whom an IQ/DQ was not measured into the two groups from all of the available information on the development of the children.

\subsection{Procedures}

The two groups were compared on the 24 variables not directly related to the key features of CDD, which were rated on a 2-point scale (present vs absent/yes vs no) with numerical data dichotomized at the relevant cutoffs. The 24 variables consisted of two before SL, one during SL (i.e. continuing for 6 months or less after onset in all of the CDD and ADSL children) and 21 at the time of first visit (i.e. an average of $5.4(\mathrm{SD}=3.8)$ and $5.0(\mathrm{SD}=$ 4.1) years after SL onset in CDD and ADSL, respectively, with no significant difference between them). Of the two variables before SL, one is the number of obstetric risk factors $\geq 3$ (above the mean of 2.4 in the 98 children in whom the risk factors were counted) from the possible total of 30 items (e.g., maternal physical diseases, threatened abortion, medication during pregnancy, cord complications, premature rupture of the membrane, cesarean section, asphyxia and severe icterus). The other is a psychosocial event before SL, which is frequently reported in cases of CDD [6,8,21] and its synonyms, Dementia infantilis [1], Heller's syndrome [33] and disintegrative psychosis [34,35]. The one variable during $\mathrm{SL}$ is fearfulness during regression, which is fairly frequently reported in cases of CDD $[8,11,14,36]$ and its synonyms [1,33-35]. The remaining 21 variables at the time of the first visit include 5 variables (i.e. having a first-degree relative with a developmental disorder (e.g., PDD, intellectual disability), epileptic EEG abnormality, epilepsy, mutism and severe intellectual disability), and 16 variables on the CARS-TV: a total score $\geq 30$ (mildly or more severely autistic) and 15 item scores $\geq 2$ on a 4-point scale (1, within normal limits; 2, mildly abnormal; 3, moderately abnormal; and 4, severely abnormal). If early-onset CDD is regarded as an extreme variant of regressive $\mathrm{AD}$, our study may be understood as a comparative study between such regressive $\mathrm{AD}$ and the rest of regressive $\mathrm{AD}$.

The second author who was not a member of the clinical teams responsible for having diagnosed CDD and ADSL extracted values of the variables directly from copies of the detailed clinical records of the children in which all of the information related to diagnoses was masked.

\subsection{Statistical Analysis}

Using IBM SPSS 20 for Windows, we compared the two 
groups of early-onset CDD and ADSL using Fisher's exact test for the 24 dichotomized variables with phi as the effect size measure of the strength of the relationship. The significance level was set at two-sided $\mathrm{p}<0.05$.

\section{RESULTS}

Table 2 shows that a psychosocial event before SL tended to be more common (a small significant effect) and fearfulness during SL was significantly more common (a large significant effect) in early-onset CDD compared with ADSL.

Table 2 also shows that epilepsy tended to be more common (a medium significant effect), CARS-TV total score $\geq 30$ tended to be less common (a small significant effect), and CARS-TV item 2 score $\geq 2$ was significantly less common (a small significant effect) in early-onset CDD compared with ADSL. There was no significant difference and effect in the remaining 19 variables: obstetric risk factors, epileptic EEG abnormality, mutism, having a first-degree relative with a developmental disorder, severe intellectual disability (the IQ/DQ did not differ significantly between the 8 early-onset CDD (mean $=34.6, \mathrm{SD}=12.0)$ and 83 ADSL (mean $=38.7, \mathrm{SD}=$ $16.3, \mathrm{t}(89)=0.693, \mathrm{p}=0.490)$, and 14 CARS-TV items.

\section{DISCUSSION}

The findings for the 24 variables not directly related to the key features of CDD should be regarded as prelimi-

Table 2. Comparison of 24 variables not directly related to the key features of childhood disintegrative disorder (CDD) between early-onset (< age 3) CDD and autistic disorder with speech loss (ADSL).

\begin{tabular}{|c|c|c|c|c|}
\hline & $\begin{array}{c}\text { Early-onset CDD } \\
(\mathrm{N}=8)\end{array}$ & $\begin{array}{c}\text { ADSL } \\
(\mathrm{N}=92)\end{array}$ & $\begin{array}{c}\text { Fisher's exact } \\
\text { test }\end{array}$ & $\begin{array}{c}\begin{array}{c}\text { Effect size } \\
\text { (phi) }\end{array} \\
\end{array}$ \\
\hline Variable & $\mathrm{n}(\%)$ & n $(\%)$ & $\mathrm{p}$ value & ${ }^{*} \mathrm{p}<0.05$ \\
\hline \multicolumn{5}{|l|}{ Before speech loss (SL) } \\
\hline Obstetric risk factors $\geq 3$ (in 90 ADSL) & $2(25.0)$ & $43(47.8)$ & 0.282 & 0.125 \\
\hline Psychosocial event before SL & $6(75.0)$ & $34(37.0)$ & 0.057 & $0.211^{*}$ \\
\hline \multicolumn{5}{|l|}{ During SL (for 6 months or less after onset) } \\
\hline Fearfulness & $5(62.5)$ & $4(4.3)$ & 0.000 & $0.551^{*}$ \\
\hline \multicolumn{5}{|l|}{ At first visit } \\
\hline Epilepsy & $2(25.0)$ & $3(3.3)$ & 0.050 & $0.271^{*}$ \\
\hline Epileptic EEG abnormality (in 7 CDD and 82 ADSL) & $2(28.6)$ & $19(23.2)$ & 0.666 & 0.034 \\
\hline Mutism & $1(12.5)$ & $27(29.3)$ & 0.436 & 0.102 \\
\hline Having a first-degree relative with a developmental disorder & $1(12.5)$ & $21(22.8)$ & 0.681 & 0.068 \\
\hline Severe intellectual disability $(\mathrm{IQ}<35)$ & $4(50.0)$ & $49(53.3)$ & 1.000 & 0.018 \\
\hline \multicolumn{5}{|l|}{ Childhood Autism Rating Scale-Tokyo Version } \\
\hline Total score $\geq 30$ & $6(75.0)$ & $88(95.7)$ & 0.072 & $0.236^{*}$ \\
\hline \multicolumn{5}{|l|}{15 items $($ score $\geq 2$ ) } \\
\hline 1. Relationships with people & $7(87.5)$ & $90(97.8)$ & 0.223 & 0.164 \\
\hline 2. Imitation & $4(50.0)$ & $76(82.6)$ & 0.049 & $0.221^{*}$ \\
\hline 3. Emotion & $8(100.0)$ & $89(96.7)$ & 1.000 & 0.052 \\
\hline 4. Use of body (stereotypy) & $7(87.5)$ & $80(87.0)$ & 1.000 & 0.004 \\
\hline 5. Relation to nonhuman objects (inappropriate interest in or use of objects) & $8(100.0)$ & $80(87.0)$ & 0.591 & 0.109 \\
\hline 6. Adaptation to environmental change & $8(100.0)$ & $81(88.0)$ & 0.593 & 0.104 \\
\hline 7. Visual responsiveness & $7(87.5)$ & $86(93.5)$ & 0.453 & 0.064 \\
\hline 8. Auditory responsiveness & $6(75.0)$ & $81(88.0)$ & 0.278 & 0.105 \\
\hline 9. Near receptor responsiveness & $5(62.5)$ & $75(81.5)$ & 0.196 & 0.129 \\
\hline 10. Anxiety & $3(37.5)$ & $56(60.9)$ & 0.267 & 0.129 \\
\hline 11. Verbal communication & $8(100.0)$ & $91(98.9)$ & 1.000 & 0.030 \\
\hline 12. Nonverbal communication & $8(100.0)$ & $91(98.9)$ & 1.000 & 0.030 \\
\hline 13. Activity level & $5(62.5)$ & $78(84.8)$ & 0.133 & 0.161 \\
\hline 14. Intellectual functioning (uneven intellectual profile) & $8(100.0)$ & $91(98.9)$ & 1.000 & 0.030 \\
\hline 15. General impressions & $8(100.0)$ & $90(97.8)$ & 1.000 & 0.042 \\
\hline
\end{tabular}


nary, due to the small number of children with earlyonset CDD. The significant difference in the two variables and its tendency in the three variables (small to large significant effects in the five variables) suggest a certain distinctiveness of early-onset CDD that sets it apart from ADSL. On the other hand, the absence of any significant difference and effect in the remaining 19 variables indicates a close similarity of early-onset CDD to ADSL. If early-onset CDD is regarded as an extreme variant of regressive $\mathrm{AD}$, our findings on the difference between early-onset CDD and ADSL can be viewed exactly as those between such a form of regressive AD and the rest of regressive AD.

The finding that fearfulness during regression was significantly more common in early-onset CDD than ADSL might reflect a hypersensitivity resulting from alteration in brain function that emerged during the course of regression is more common in early-onset CDD than ADSL. A recent study [36] reported a high frequency of such fearfulness (defined as terror/agitation preceding regression in the study) in Heller's original cases and also in the authors' experience with 20 or more cases of CDD. The study highlights the importance of this phenomenon for further investigation, both as a diagnostic descriptor of CDD and a clue to the mechanism of autistic regression. The tendency of higher incidence of epilepsy in early-onset CDD than ADSL may also suggest that more severe brain dysfunction underlies early-onset CDD compared with ADSL. The tendency of a higher incidence of psychosocial events before regression in early-onset CDD than ADSL might suggest that a certain vulnerability of brain function before regression is greater in early-onset CDD than ADSL. Such events that occur before regression need verification with a reliable and valid life event scale.

The discrepancy between the short-term outcome not being significantly different between early-onset CDD and ADSL in this study based on children and the longterm outcome having been shown to be worse in CDD than autism in previous studies [6,7] based on adolescents and adults, may be accounted for by the fact that mental development slows with age more profoundly in CDD than in autism. Such intellectual decline over time is suggested by the finding in a study [37] that the number of persons with an IQ under 50 increases at a greater rate from infancy to the late $20 \mathrm{~s}$ in disintegrative psychosis, a synonym of CDD (from $54 \%$ to $92 \%$ ), than in autism (from $46 \%$ to $61 \%$ ).

Early-onset CDD may be less autistic than ADSL in childhood, as suggested by the tendency to milder autism and significantly milder impairment in imitative skills on the CARS-TV. This finding, however, appears contrary to the finding that CDD has significantly more autistic symptoms than autism in a previous study [7]. This dis- crepancy may be accounted for in part by the difference between the autism groups. We employed only ADSL which is a condition that is more severely impacted in the degree of intellectual disability, which is known to correlate with that of autism [29,30], than non-regressive autism [22-25], while the other study [7] employed the whole range of autism (regressive and non-regressive included) considered to have a milder degree of intellectual disability and thus a milder degree of autism than regressive autism. In addition, a milder degree of autism in early-onset CDD than ADSL in childhood may be reversed in adolescence/adulthood. This is because there is a possibility that cases of CDD will develop more severe intellectual disability with increasing age than autism [37] and thus there are more profound autistic symptoms, since the severity of intellectual disability correlates with the severity of autism [29,30].

The findings in this study are based on a small number of children with early-onset CDD, so they need to be tested more extensively in a multicenter study on a larger number of children with early-onset CDD. Furthermore, comparisons between early-onset CDD and late-onset $(\geq$ age 3) CDD, and between late-onset CDD and regressive $\mathrm{AD}$ are needed to investigate the exact character of CDD more precisely. Such comparisons would be better performed in a study of longitudinal rather than cross-sectional design.

In conclusion, the occurrence of regression regardless of its severity in infancy and the absence of significant difference found for the great majority of the variables not related to the key features of CDD between earlyonset CDD and ADSL support subsuming the entire part of CDD under regressive ASD, while the significant difference found for a small number of the variables between the two conditions warrants studying CDD as a prototypical form of regressive ASD.

\section{REFERENCES}

[1] Heller, T. (1908) Über Dementia infantilis: Verblödungsprozess im Kindesalter. Zeitschrift für die Erforschung und Behandlung des Jugendlichen Schwachsinns, 2, 1728.

[2] American Psychiatric Association (1994) Diagnostic and statistical manual of mental disorders. 4th Edition, American Psychiatric Association, Washington DC.

[3] World Health Organization (1993) The ICD-10 classification of mental and behavioural disorders: Diagnostic criteria for research. World Health Organization, Geneva.

[4] Fombonne, E. (2009) Epidemiology of pervasive developmental disorders. Pediatric Research, 65, 591-598. doi:10.1203/PDR.0b013e31819e7203

[5] Malhotra, S. and Gupta, N. (2002) Childhood disintegrative disorder: Re-examination of the current concept. European Child \& Adolescent Psychiatry, 11, 108-114. 


\section{doi:10.1007/s00787-002-0270-6}

[6] Volkmar, F.R. and Cohen, D.J. (1989) Disintegrative disorder or "late onset" autism. Journal of Child Psychology and Psychiatry, 30, 717-724.

doi:10.1111/j.1469-7610.1989.tb00784.x

[7] Volkmar, F.R. and Rutter, M. (1995) Childhood disintegrative disorder: Results of the DSM-IV autism field trial. Journal of the American Academy of Child \& Adolescent Psychiatry, 34, 1092-1095. doi:10.1097/00004583-199508000-00020

[8] Kurita, H., Osada, H. and Miyake, Y. (2004) External validity of childhood disintegrative disorder in comparison with autistic disorder. Journal of Autism and Developmental Disorders, 34, 355-362. doi:10.1023/B:JADD.0000029556.25869.71

[9] Kurita, H., Koyama, T., Setoya, Y., Shimizu, K. and Osada, H. (2004) Validity of childhood disintegrative disorder apart from autistic disorder with speech loss. European Child \& Adolescent Psychiatry, 13, 221-226. doi:10.1007/s00787-004-0397-8

[10] American Psychiatric Association (2012) DSM-5 autism spectrum disorder news release 1-20-12. http://www.dsm5.org

[11] Jaydeokar, S., Bal, G. and Shah, N. (1997) Childhood disintegrative disorder: A case report. Indian Journal of Psychiatry, 39, 85.

[12] Burd, L., Ivey, M., Barth, A. and Kerbeshian, J. (1998) Two males with childhood disintegrative disorder: A prospective 14-year outcome study. Developmental Medicine \& Child Neurology, 40, 702-707. doi:10.1111/j.1469-8749.1998.tb12331.x

[13] Zwaigenbaum, L., Szatmari, P., Mahoney, W., et al. (2000) Case report: High functioning autism and childhood disintegrative disorder in half brothers. Journal of Autism and Developmental Disorders, 30, 121-126. doi:10.1023/A:1005455505211

[14] Agarwal, V., Sitholey, P. and Mohan, I. (2005) Childhood disintegrative disorder, an atypical presentation: A case report. Journal of Autism and Developmental Disorders, 35, 873-874. doi:10.1007/s10803-005-0033-6

[15] Volkmar, F.R., Koenig, K. and State, M. (2005) Case report. In: Volkmar, F.R., Paul, R., Klin, A. and Cohen, D. Eds., Handbook of Autism and Pervasive Developmental Disorders, 3rd Edition, John Wiley \& Sons Inc., Hoboken, 83-84.

[16] Palomo, R., Thompson, M., Colombi, C., et al. (2008) A case study of childhood disintegrative disorder using systematic analysis of family home movies. Journal of Autism and Developmental Disorders, 38, 1853-1858. doi:10.1007/s10803-008-0579-1

[17] Mordekar, S.R., Prendergast, M., Chattopadhyay, A.K. and Baxter, P.S. (2009) Corticosteroid treatment of behaviour, language and motor regression in childhood disintegrative disorder. European Journal of Paediatric Neurology, 13, 367-369. doi:10.1016/j.ejpn.2008.06.001

[18] Homan, K.J., Mellon, M.W., Houlihan, D. and Katusic, M.Z. (2011) Brief report: Childhood disintegrative disorder: A brief examination of eight case studies. Journal of
Autism and Developmental Disorders, 41, 497-504. doi:10.1007/s10803-010-1063-2

[19] Charan, S.H. (2012) Childhood disintegrative disorder. Journal of Pediatric Neurosciences, 7, 55-57. doi:10.4103/1817-1745.97627

[20] Malhotra, S., Subodh, B. N., Parakh, P. and Lahariya, S. (2013) Brief report: Childhood disintegrative disorder as a likely manifestation of vitamin B12 deficiency. Journal of Autism and Developmental Disorders, Epub ahead of Print. doi:10.1007/s10803-013-1762-6

[21] Rosman, N.P. and Bergia, B.M. (2013) Childhood disintegrative disorder: Distinction from autistic disorder and predictors of outcome. Journal of Child Neurology, Epub ahead of Print. doi:10.1177/0883073812472391

[22] Kurita, H. (1985) Infantile autism with speech loss before the age of thirty months. Journal of the American Academy of Child Psychiatry, 24, 191-196. doi:10.1016/S0002-7138(09)60447-7

[23] Hoshino, Y., Kaneko, M., Yashima, Y., et al. (1987) Clinical features of autistic children with setback course in their infancy. Psychiatry and Clinical Neurosciences, 41, 237-245. doi:10.1111/j.1440-1819.1987.tb00407.x

[24] Kobayashi, R. and Murata, T. (1998) Setback phenomenon in autism and long-term prognosis. Acta Psychiatrica Scandinavica, 98, 296-303.

doi:10.1111/j.1600-0447.1998.tb10087.x

[25] Bernabei, P., Cerquiglini, A., Cortesi, F. and D'Ardia, C. (2007) Regression versus no regression in the autistic disorder: Developmental trajectories. Journal of Autism and Developmental Disorders, 37, 580-588. doi:10.1007/s10803-006-0201-3

[26] Baird, G., Charman, T., Pickles, A., et al. (2008) Regression, developmental trajectory and associated problems in disorders in the autism spectrum: The SNAP study. Journal of Autism and Developmental Disorders, 38, 1827 1836. doi:10.1007/s10803-008-0571-9

[27] World Health Organization (1977) International classification of diseases. 1975 Revision, Vol. 1, World Health Organization, Geneva.

[28] American Psychiatric Association (1980) Diagnostic and statistical manual of mental disorders. 3rd Edition, American Psychiatric Association, Washington DC.

[29] Kurita, H., Miyake, Y. and Katsuno, K. (1989) Reliability and validity of the Childhood Autism Rating ScaleTokyo Version (CARS-TV). Journal of Autism and Developmental Disorders, 19, 389-396. doi:10.1007/BF02212937

[30] Schopler, E., Reichler, R.J., DeVellis, R.F. and Daly, K. (1980) Toward objective classification of childhood autism: Childhood Autism Rating Scale (CARS). Journal of Autism and Developmental Disorders, 10, 91-103. doi:10.1007/BF02408436

[31] Koyama, T., Osada, H., Tsujii, H. and Kurita, H. (2009) Utility of the Kyoto Scale of Psychological Development in cognitive assessment of children with pervasive developmental disorders. Psychiatry and Clinical Neurosciences, 63, 241-243. doi:10.1111/j.1440-1819.2009.01931.x 
[32] Kurita, H., Osada, H., Shimizu, K. and Tachimori, H. (2003) Validity of DQ as an estimate of IQ in children with autistic disorder. Psychiatry and Clinical Neurosciences, 57, 231-233.

doi:10.1046/j.1440-1819.2003.01106.x

[33] Kurita, H. (1988) The concept and nosology of Heller's syndrome: Review of articles and report of two cases. Psychiatry and Clinical Neurosciences, 42, 785-793. doi:10.1111/j.1440-1819.1988.tb01167.x

[34] Evans-Jones, L.G. and Rosenbloom, L. (1978) Disintegrative psychosis in childhood. Developmental Medicine \& Child Neurology, 20, 462-470. doi:10.1111/j.1469-8749.1978.tb15247.x

[35] Kurita, H., Kita, M. and Miyake, Y. (1992) A compara- tive study of development and symptoms among disintegrative psychosis and infantile autism with and without speech loss. Journal of Autism and Developmental Disorders, 22, 175-188. doi:10.1007/BF01058149

[36] Westphal, A., Schelinski, S., Volkmar, F. and Pelphrey, K. (2013) Revisiting regression in autism: Heller's Dementia infantilis: Includes a translation of Über Dementia Infantilis. Journal of Autism and Developmental Disorders, 43, 265-271. doi:10.1007/s10803-012-1559-z

[37] Mouridsen, S.E., Rich, B. and Isager, T. (1998) Validity of childhood disintegrative psychosis: General findings of a long-term follow-up study. British Journal of Psychiatry, 172, 263-267. doi:10.1192/bjp.172.3.263 